\title{
Respiratory syncytial virus (RSV) in pediatric influenza-like illness (ILI) cases in Lombardy, Northern Italy, during seven consecutive winter seasons (from 2014-2015 to 2020-2021)
}

\author{
Laura Pellegrinelli ${ }^{1}$, Cristina Galli ${ }^{1}$, Laura Bubba ${ }^{1}$, Arlinda Seiti ${ }^{1}$, Giovanni Anselmi ${ }^{1}$, \\ Valeria Primache ${ }^{1}$, Lucia Signorini ${ }^{1}$, Serena Delbue ${ }^{1}$, Sandro Binda ${ }^{1}$, and Elena Pariani ${ }^{1}$ \\ ${ }^{1}$ University of Milan
}

October 24, 2021

\begin{abstract}
INTRODUCTION. Respiratory syncytial virus (RSV) is the major cause of lower respiratory tract illness in young children and can also cause influenza-like illness (ILI). Here we investigated the epidemiological features of RSV infection in pediatric ILI cases in Lombardy (a region in Northern Italy accounting nearly 10-million inhabitants) from 2014-2015 to $2020-2021$ winter seasons. MATERIAL AND METHODS. Data for this study were retrieved and statistically analyzed from the database of virological influenza surveillance of the regional reference laboratory for Lombardy within the Italian influenza surveillance network (InfluNet). RESULTS. RSV accounting for nearly $19 \%$ of pediatric ILI with a risk of infection nearly 2 -fold greater than that of individuals [?]15 years. The RSV positivity rate increased to $28 \%$ considering $0-5$ years old children. Although in children [?] 5 years the risk of infection from influenza viruses resulted nearly 2-fold higher than the risk of RSV infection, the age group 4-6 months and 7-12 months showed 5-fold greater risk of infection from RSV than from influenza. Children [?]5 years of age with presence of one or more comorbidities had a nearly 5-fold greater risk of getting RSV infection than otherwise healthy 0-5 years old children. DISCUSSION. The use of the ILI sentinel surveillance allowed us to identify groups at higher risk of RSV and influenza infection and to define the start, duration, timing and intensity of the RSV and influenza community circulation, determining thresholds based on historical data. This surveillance approach can be implemented to assess the nearly real-time RSV circulation and impact.
\end{abstract}

\section{Hosted file}

RSV_Final.docx available at https://authorea.com/users/442693/articles/542893-respiratorysyncytial-virus-rsv-in-pediatric-influenza-like-illness-ili-cases-in-lombardy-northernitaly-during-seven-consecutive-winter-seasons-from-2014-2015-to-2020-2021 